\title{
Specification and execution of tests using tMSC
}

\author{
Iulian Ober \\ INPT-ENSEEIHT, 2 rue Camichel, 31000 Toulouse cedex, France \\ e-mail: Iulian.Ober@enseeiht.fr
}

\author{
Alain Kerbrat \\ Verilog, 150 rue Nicolas Vauquelin, BP1310, 31106 Toulouse cedex, France \\ e-mail: Alain.Kerbrat@verilog.fr
}

Key words: conformance testing, test execution, Msc, TTCN, TCL

Abstract: Writing tests for concurrent systems is difficult and time consuming. Handling concurrency requires an expressive language, yet the same language should be easy to read and edit. A standardized test description language such as TTCN is often perceived as too cryptic, difficult to read and generally too powerful. Other test description languages are home made and do not allow to share or to maintain easily existing test suites. We present a test specification and execution tool relying on MSC. The Msc language is standardized, and provides a graphical and intuitive way for describing test cases.

\section{INTRODUCTION}

The validation phase is the most time consuming part of software development. It is especially true for telecommunications systems, where any new software must integrate smoothly and quickly with existing ones. The validation of a new telecommunication service can take 5 to 10 times the time needed to develop it. Validation is also a major concern for safety

* work supported by the CS Communications \& Systems Group 
critical systems such as embedded systems in planes, satellites or nuclear plants. These systems need to be certified, hence must be validated extensively and test cases must be traced from the requirements down to the code.

Several test description languages exist for concurrent and safety critical systems. TTCN [1] is a standardized test description language, meant for conformance testing [2]. It is a powerful language, combining constructs for the description of the behavior of test cases and operators for building concurrent test cases. It integrates ASN.1 [6] as well as its own data type language and allows complex descriptions for data values. However, this same expressive power, combined with its difficult syntax and presentation requirements, hampers TTCN use. TTCN test cases are sometimes hard to write and difficult to read.

Other test description languages are usually developed for internal purposes, as a front end of the test platform. These solutions are usually well mastered, as they are adapted to the specific needs of both the test platform and of the software developed on it. However, the semantics of these languages are usually not formally defined. This complicates the sharing of test suites, even among teams of the same company. The evolution of the languages and of their compilers is usually done on a per project basis, which makes it difficult to maintain the test suites. Last but not least, they seldom come with a graphical counterpart, as this is usually time and money consuming to develop. MSC is an interesting candidate to alleviate these problems. It is graphical, allowing for intuitive reading or editing of a test case behavior. It is also an international standard [8], which evolves steadily every four years.

To meet the need for a simpler yet not less powerful method for describing and executing conformance tests, we propose an alternative approach: the use of MSC for describing test cases, complemented with a second-level formalism - the Test Description Language (TDL), all within a lightweight and open framework designed for executing tests on a wide class of possible systems. The MSC is a language for expressing execution traces that has been used for a long time for capturing requirements in telecommunication systems design but also as a basis for system simulation and validation, interface specification, etc. It is a standard and mature graphical language, usable for expressing test cases at a first level, and whose simplicity appeals.

This paper advocates our testing framework, by showing the strong and weak points of MSC when expressing tests, by proving that they can be overcome by using a second-level formalism for expressing test cases (the Test Description Language) and by describing the architecture of a tool 
implementing our testing paradigm and emphasizing on the open parts which make the tool usable in many different contexts.

Organization of the paper. The first part of this paper presents tMSC. tMSC is the subset of MSC we chose for test cases description.

The second part describes the TestPlayer tool. TestPlayer is a tool for edition, and execution of test cases written in tMSC. TestPlayer provides also a tool to visualize back at the MSC level the test execution results.

The third part describes the connection of TestPlayer with a related tool, TestComposer. TestComposer allows the automated generation of test cases in tMSC, from a SDL description.

\section{USING MSC FOR DESCRIBING TEST CASES}

MSC is a visual language for representing system execution traces, mainly in terms of messages exchanged between the internal instances of a system and with the environment. It is being standardized by the International Telecommunication Union as Recommendation Z.120 [8]. The standardization work began in 1990 and each four years a new major version is issued.

Being visual, MsCs are more easily readable, which makes them more attractive to the users. Thus there were early attempts to use MSC for expressing test cases or test purposes [4]. MSCs are suitable for this purpose if we bear in mind that the behavioral part of a TTCN test case describes also a set of possible traces of messages exchanged between the system and its environment, each such trace having associated a verdict at the end.

These attempts have shown the benefits, but also the weaknesses of MsCs when expressing test cases: there is no notion of test verdict in MSC, no possibility to specify data types and constraints on data, no possibility to use variables and no notion of test architecture [3]. We will see in what follows how we overcame these problems in our approach with TestPlayer.

\subsection{MsCs for test}

To use MSCs to represent test cases, we must give an intuitive meaning to test MSCs in this context (denoted tMSC in what follows). A conformance test for a protocol [2] is expressed in terms of the messages exchanged between the tester and the Points of Control and Observation (PCOs) of the system. (a PCO is a named interface through which the system receives or sends signals [2]).

We distinguish three flavors of test MSCs differentiated by the instances they represent. The first one (see Figure 1) takes the point of view of the 
tester: it represents instances denoting PCOs and testers (one or more). The second variety of tMSCs takes the point of view of the system, containing an instance that represents the system and one instance for each PCO. Abstract test description for standard protocols often employ this kind of MSCs. Finally, usual MSCs are acceptable as test MSCs, case in which the tester is represented by the environment. This kind of MSCs is likely to result from the simulation of an SDL model, for example.

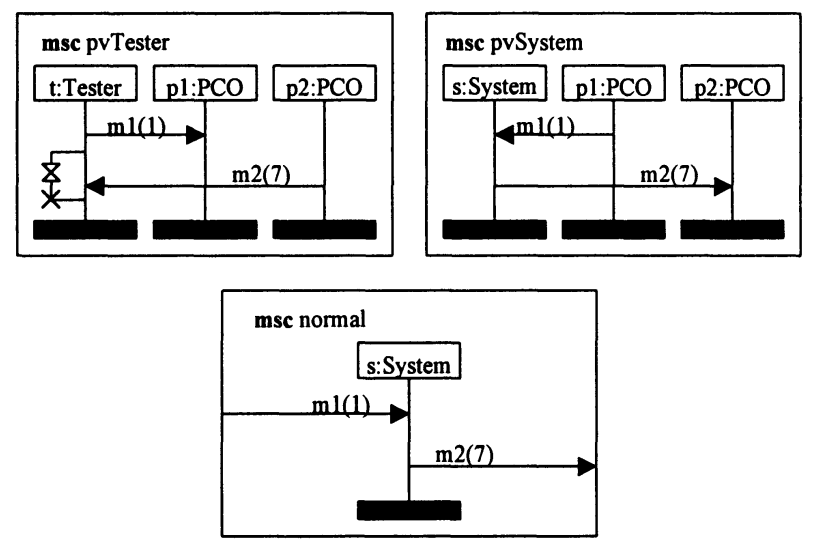

Figure 1. The three varieties of test Mscs

Usually a MSC describes one possible behavioral trace of a system, be it complete or incomplete. We consider a different semantics for the MsCs used for describing test cases: a test MSC contains the main test sequence, i.e. the sequence of events leading to the success of the test (PASS verdict). All deviation from this sequence (a message that should but cannot be sent, a message that should be received but is not) produces a failure of the test.

One inconvenient when using MSCs for describing test cases is that one cannot express constraints on the parameters of the messages (see also [3]). To alleviate this limitation, we defined a set of generic specifiers that may be used in MSCs: AnyValue, ValueList, and ValueRange. Still, the amount of constraints we are able to express using these specifiers in MSC is limited, due to the lack of variables. This is one reason for which TestPlayer uses a second language for describing tests, TDL (see section 3.2).

Besides messages, three additional categories of constructs have a meaning on a Basic tMSC, but only when they appear on a tester instance: stop, test timers and coregions.

- A stop construct on a tester bar means that the test stops immediately with a PASS verdict. 
- Timer constructs (set/reset/timeout) are used to express minimal/maximal delay bounds. A timer set followed by a timeout represents a lower bound, a timer set followed by a reset represents an upper bound (this follows from the assumption that the MSC represents the main sequence that leads to a PASS verdict). The timing constraints employed in test cases usually take the following form: the time span between the event a and the event $\mathrm{b}$ must be between $\mathrm{x}$ and $\mathrm{y}$ time units. The way of expressing this with timers in shown in Figure 2.

- A coregion on a tester bar means that the messages that come from the system (PCOs) within the boundaries of the coregion may come in any order. As a difference from Z.120, only response messages coming from the system and timer timeouts may be represented within a coregion. This is because the tester must be controllable, so we cannot allow message outputs and other actions for which we do not specify the precise order.

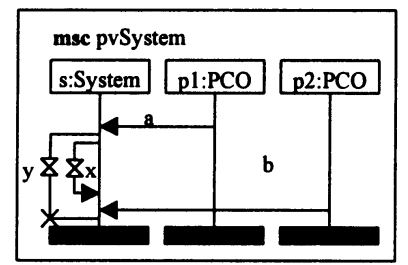

Figure 2. Delay intervals expressed with timers

\subsection{Composing test MsCs}

MSC'96 defines a set of structural composition operators for organizing tMsCs into High-Level tMSCs. We use a subset of them to express tests. For their correct composition, two MSCs must define the behavior of the same tester(s) on the same system (PCOs). The semantics of these composition operators follows the MSC'96 standard:

- The sequential composition of two tMSCs indicates that the tester first executes the constructs specified by the first MSC and then, if it did not fail, executes the constructs specified by the second MSC.

- The repetition of a tMSC indicates that the tester repetitively executes the constructs specified by the MSC.

- The alternative composition of two tMsCs indicates that the tester executes either the constructs specified by the first MSC or the constructs specified by the second MSC. The choice is made between the first constructs from the two tMSCs, which must be a message receipt or a timer timeout . 
We do not support other MSC'96 operators such as parallel composition and exception as they would produce non controllable test cases.

\subsection{Expressive power of the tMsc. tMsC vs. TTCN}

Not all the three flavors of test MSCs have the same power of expression: only tMSCs that take the point of view of the tester support all the operators defined in section 2.1 (ex. timers may appear only on tester instances).

The Tree and Tabular Combined Notation (TTCN [1]) is the ISO/IEC standard language for the description of abstract test suites. TTCN was designed with the ISO layered model as a framework and it is specialized for conformance testing.

Being a complete test language, TTCN brings more power than tMSCs. TTCN supports data and signal types definition, variables, complex expressions on expected values, concurrent testers, explicit and more accurate test verdicts. On the behavior description side, TTCN offers many constructs for expressing concurrency and capturing non determinism (through the use of INCONCLUSIVE verdicts). Our experience is, however, that concurrent and non-deterministic test cases are usually difficult to write. Thus, TTCN test cases often are reduced to deterministic, single tester sequences, which falls back within the expressive power of tMsCs.

The one important advantage of using MSCs to express test cases is that MSC is a visual and intuitive. MSC was used for several years in the definition of protocol standards and one can derive test MSCs straight from the MSC coming from the protocol standard.

Using the semantics outlined above, tMsC is able to express the same behaviors as TTCN. MSC timers may express everything that is described in terms of timers in TTCN. MSC composition operators make it possible to express branching behavior in the same way as in complex TTCN trees.

However, to cover the lack of variables and data definitions, we use a second language, TDL, which is the internal language of TestPlayer. We decided to make this language visible to the user, while waiting for the future standard MSC2000 to cover some of the mentioned lacks of tMSC.

\section{TESTPLAYER: AN OPEN TEST PLATFORM}

TestPlayer is an open architecture for testing providing customizability and power of expression sufficient to be applicable to a large number of real systems and test cases.

In our model, several testers feed the IUT with signals and check the responses of the system, and in the end generate test verdicts that will be 
logged in a results database. The interfacing between the IUT and the testers is done at predefined PCOs. The mechanisms through which messages are physically passed to the system depend on the system internals, so the testers are modularized such that the communication details are separated from the behavior description of the tester.

The behavior description of a tester is in fact a high level piece of code written in our Test Description Language (TDL), which regards signals as abstract data structures (is not concerned with bit-level encoding) and identifies PCOs only through their name (regardless of the details of physical communication with a specific interface point of the system). All these communication/encoding/decoding details are implemented as modules of the TDL interpreter and are customizable, as we will see in more detail later.

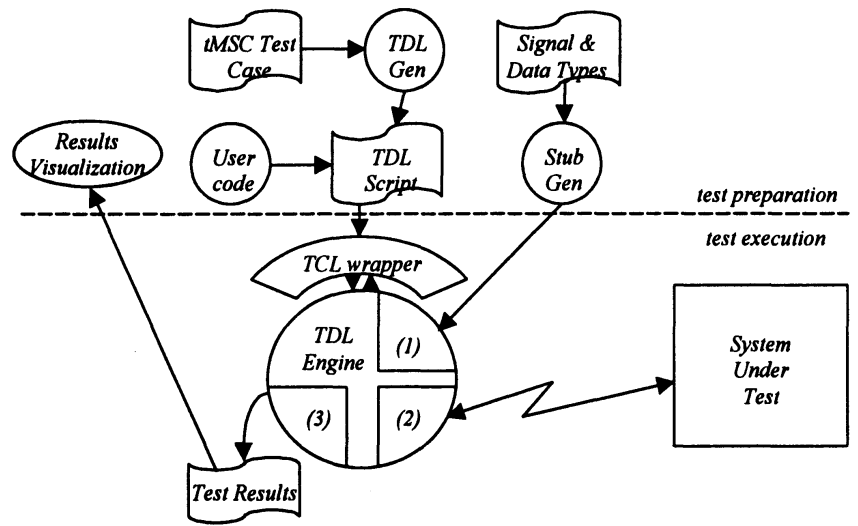

Figure 3. TestPlayer Architecture

Testing a system with TestPlayer is a two-phase process (see Figure 3): there is a preparation phase and an execution phase. The source of the test case may be either a tMSC diagram or a hand written TDL script. In the first case, what we will do is to automatically translate the tMSC in TDL using a MSC-to-TDL compiler (TDL Gen on Figure 3).

Before executing the tests the user must provide the description of the signals and data types exchanged between the tester and the system. This information is needed at execution time by the component in charge with data encoding/decoding ((1) on Figure 3). The user may either provide directly the body of the component (1) or - more likely - provide a SDL or ASN.1 description of the signals and data types. If the latter is the case, the body of component (1) is generated automatically by a sort of compiler (Stub Gen on Figure 3).

At execution time, the core of the TestPlayer environment is the TDL execution engine - the implementation of the TDL primitives. This is a fixed 
part of the architecture i.e. it cannot be customized. The implementation of the test primitives in the TDL engine is a static library that may be used from a variety of host languages such as $\mathrm{C}, \mathrm{TCL}$, Pascal. For each host language there must be a wrapper that makes the primitives accessible from the language.

The most common case of usage of TestPlayer is with the TCL interface. Therefore the test primitives are available as TCL commands. From now on, TDL will denote the extended TCL language.

\subsection{TestPlayer customizable components}

The TDL engine uses a set of utility components, which may be customized by the user. The user is free to provide any replacement for a component, provided he follows a set of simple naming conventions.

The Signal Encoding/Decoding component denoted by (1) on Figure 3 contains callback functions for signal and data encoding/decoding/matching. These functions are called to pack/unpack the signals and data transmitted/ received over the communication line that links the tester and the system. Component (1) also contains the functions that match an incoming message towards a generic message specification. As mentioned above, the Signal Encoding/Decoding component may either be provided as such by the user, or it may be generated from the SDL or ASN.1 description of the system signals and data types.

The Protocol component denoted by (2) on Figure 3 implements the protocol used by the tester to communicate with the system under test. TestPlayer may be used to apply tests in different configurations, in which the tester and the SUT may be on the same machine or on two different machines connected by a network, a serial line, etc. To allow this flexible architecture, the communication functionality must be kept in a separate component in order to allow for changes in the underlying communication primitives. The Protocol component (2) must implement a simple interface that basically knows how to open and close connections, raw send binary data to a connection and do blocking reads on a connection. Beyond this, the user is free to use whatever suitable means to implement the communication between the tester task and the SUT (TCP/IP, some proprietary protocol, OS IPC, etc.)

The Test Reports component (3) formats and stores the partial or final test results in a log. A user may require that the test results be stored in a specific format: a database, a log file with a proprietary format, etc. Any replacement for this component must implement a simple interface containing the following functionality: opening/closing a log, writing a test event to a log, writing a test verdict to a log. 
After the execution of a test case or suite, the results may be visualized and one may automatically find the initial MSC construct corresponding to a test event that appears in the log, using the Results Visualization tool.

Thus we have obtained a testing architecture based on tMSCs and TDL, but in which the parts describing system signals and data types, the communication protocol between the tester and the system, and the formatting of test results are left open and customizable.

\subsection{The Test Description Language}

The Test Description Language is the internal language of TestPlayer. There is no test execution engine in TestPlayer that takes tMSCs and executes them directly. Instead a tMSC is translated in a TDL script. At this level the programmer may edit and extend the TDL test cases.

The Test Description Language is formed of a set of primitives with a precise semantics and an implementation that may be embedded in any language that allows for extensions $(\mathrm{C}, \mathrm{TCL}$, Python, etc.). The primitives correspond to TTCN executable instructions and to certain MSC constructs. They differ from MSC in that they are imperative rather than declarative. They provide for the missing parts of tMsC, notably variables and verdicts, as well as constructs for expressing alternatives between behaviors. A complete description of TDL primitives can be found in [10].

\subsection{Translating tMsCs into TDL scripts}

For each tester described in a tMSC, one script is generated. Usually a tMSC describes only one tester, but in the case of tMSCs taking the point of view of the tester (see Figure 1) there may be more. Tester outputs are translated into TDL Output statements, tester inputs into Expect statements and so on. An example of translation for a Basic MSC is given in Figure 4.

For High-level MSCs (see Figure 5), the sequential composition of MSCs causes the concatenation of the scripts described by the operand MSCs. The alternative composition of MSCs is translated using the TDL Alternative statement and repetitive composition is translated using the repetitive construct from TCL. 


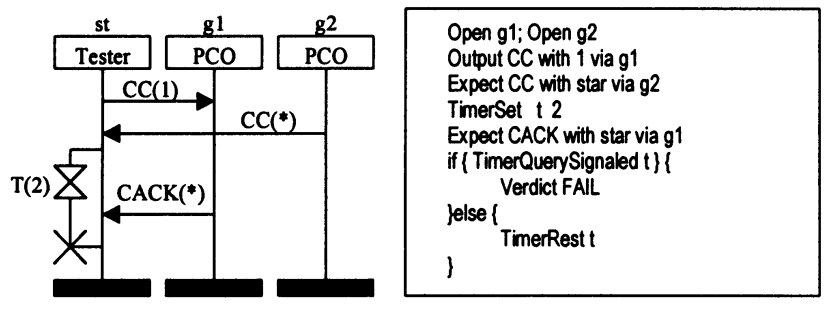

Figure 4. tMsc translated in TDL

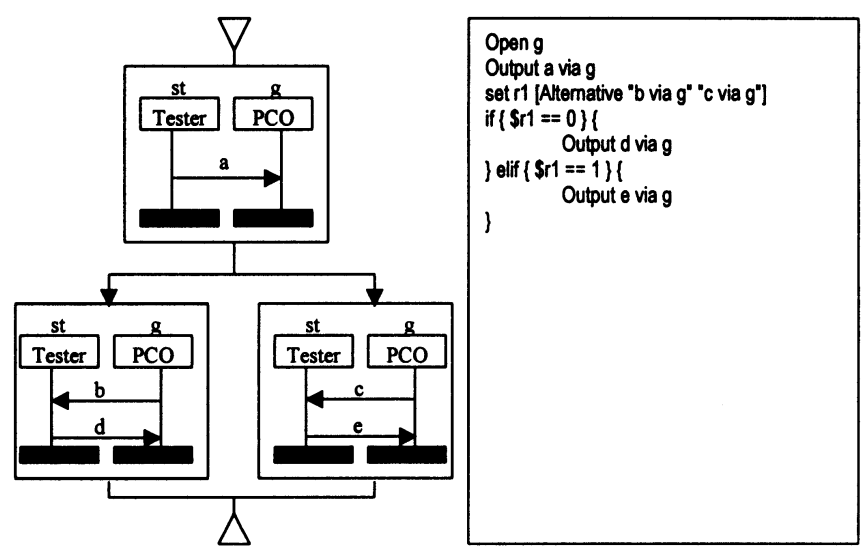

Figure 5. High-level MSC translated in TDL

\subsection{TDL benefits}

The TDL primitives bring variables and the alternative and repetitive statements. TDL primitives are implemented as an extension to the TCL language but they may be integrated in another language like $\mathrm{C}, \mathrm{C}++$, Python or Java.

Using a small set of primitives integrated in a scripting language ensures a small learning curve. TDL scripts being directly interpreted by TestPlayer, usual programming techniques like step-by-step execution and breakpoints may be employed for the execution of a test script.

\subsection{TestPlayer for ObjectGEODE}

TestPlayer for ObjectGEODE is an instantiation of all the generic components and tools described in the previous sections, which work with systems modeled in SDL [7] with ObjectGEODE [9] and for which code was 
automatically generated with the ObjectGEODE SDL-C Code Generator. The concrete architecture after the instantiation is outlined in Figure 6 (the instantiated tools and components are drawn on gray background).

The provided Stub Gen (Figure 3 and 6) is a compiler that automatically generates the code for the component (1) from the SDL description of the system. The generated component (1) contains encoding/decoding functions for the signals and data types defined in the SDL system.

Component (2) is in charge with the communication between the tester and the system. The instantiation uses TCP/IP for this purpose. An adapter task has to be added on the system side which "understands" the same protocol as the tester.

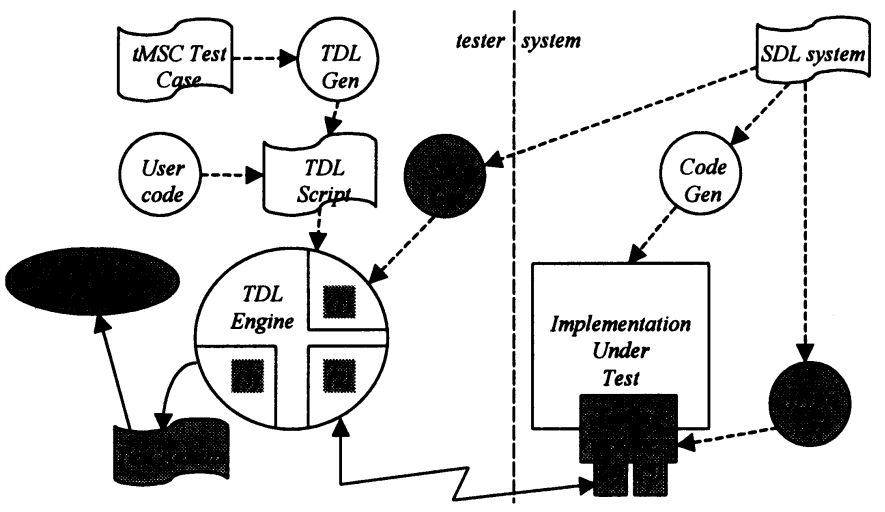

Figure 6. TestPlayer Concrete Architecture for ObjectGEODE

The adapter ("testing wrapper" on Figure 6) task will become the environment task of the system (see [9] for details). It takes the signals emitted by the system and send them over the network connection and vice versa. It exposes an interface of the system organized in PCOs. Concretely, this means that the external task is a TCP server that accepts one TCP connection for each exposed PCO. A tester that wants to communicate with the system on a specific PCO must call the Open primitive first.

The testing wrapper contains two sub-components: (4) which implements the communication protocol (very similar to (2) on the tester side) and (5) which implements signal and data types encoding/decoding (similar to (1) on the tester side). There is an automatic generator that generates the task from the SDL system specification.

The results formatting and storage component (3) is also instantiated for ObjectGEODE. It stores the test events and verdicts in an ASCII format in the test results repository. A visualization tool can read the repository and make the link back to the MSC source of a test case. 
The instantiation of the generic components showed that we can easily obtain an executable architecture useful on large class of SDL systems.

\subsection{An example}

We validated the prototype instantiation described in the previous section on several examples. This section shows how we can test a sample system with the TestPlayer prototype.

We consider a simple SDL system describing a cards game server. The high-level architecture of the system is shown in Figure 7 (for brevity, not all the signals are shown).

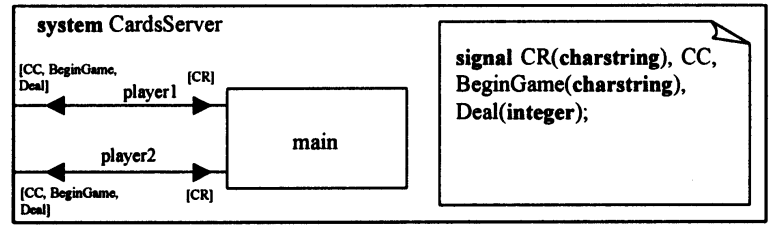

Figure 7. SDL system for a card game server

We would like to test the connection phase of the game. The connection involves both players that may connect to a game, so the tester will have to take the place of both of them. Each channel linking the system and the environment will correspond to a PCO. For testing the connection phase we will re-use a MSC from the requirements specification (Figure 8)

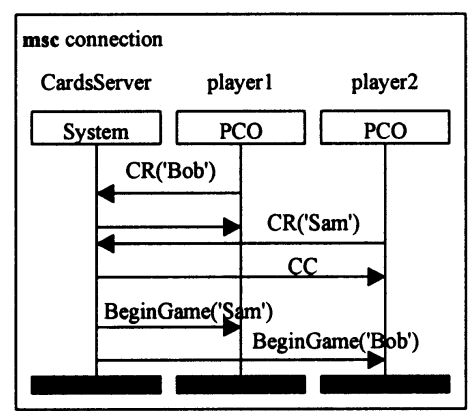

Figure 8. Connection phase of the Cards Game

After providing all the configuration parameters needed by the ObjectGEODE SDL-C Code Generator, we generate the executable system and the testing wrapper task in one pass, by invoking st_gen_sys Cardsserver. 
By default this will generate the executable system (CardsServer.exe) containing also the testing wrapper, generated in such a way that each channel linking the system and the environment becomes a PCO. This configuration suits our purpose. Other configurations are possible, like for instance one PCO for the entire system (grouping all the channels).

We now generate the extended TCL interpreter containing also the signal and type information specific to our system, by invoking st_gen_interp CardsServer. The starting point is again the SDL specification.

The generated interpreter (called tdlsh) contains the TDL engine plus components (1), (2) and (3) (see Figure 6). The type information contained in the SDL system is used for generating component (1). The other components are not rebuilt, they are taken from a library and linked together.

We have two possibilities for using the executable system and the TDL interpreter: either we generate the TDL script from the MSC(s) and execute it in batch mode, or we try an interactive session in which we feed whatever TDL commands we want to the command prompt of the TDL interpreter.

The first alternative involves first of all generating the TDL script from the MsC description of the test case. We can do this with st_gen_tests connection.msc and we obtain the TDL script called CardsServerl.tdl containing the following code (analogously to the one in Figure 4):
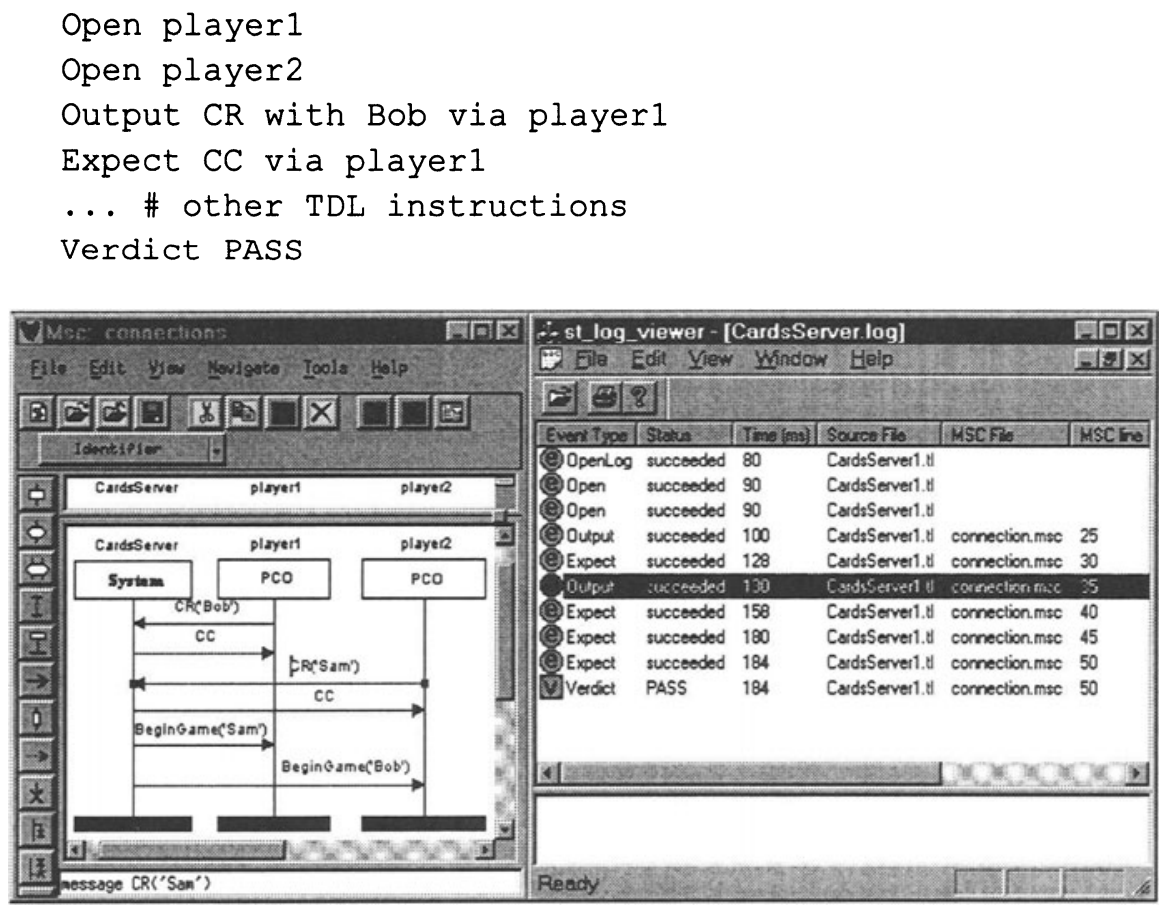

Figure 9. The Results Visualization Tool 
To run this test, we must first start the system (CardsServer.exe) in a console, and then invoke st_exec_tests CardsServerl.tdl in another console. This command will run the test in batch mode and will write the test events and verdict in Cardsserver. log.

This log may be visualized with st_view_log and the MSC construct corresponding to each event appearing in the log may be highlighted automatically in the ObjectGEODE MSC editor (see Figure 9). For FAIL verdicts, the failure reason is also stored in the log.

For using the TDL interpreter in interactive mode, one must start the system in a console and the tdlsh in another console. Then, at the tdlsh prompt he may type commands like the ones generated from the MSC. The outputs and verdicts of the commands will appear on the console.

\section{TESTPLAYER WITH TESTCOMPOSER}

TestComposer is a tool for automated test generation [5]. Starting from a SDL specification, it generates test suites for conformance testing.

TestComposer works in three phases :

- A test purposes phase, where informal test purposes coming from the requirements are written down in a formal way, using MSCs. These test purposes can also be generated automatically from the SDL specification, according to the structural coverage of the specification. In this mode, each transition (in fact basic block) of the SDL specification will be covered by at least one test purpose.

- A test cases computation phase, where a complete tree of possible behaviors is derived from each test purpose. These test cases are gathered in an internal database.

- A test suite production phase, where the test cases database is first processed in order to structure test cases, and to share common sub parts. The test suite is then written down using the TTCN language.

The test purposes used by TestComposer can either be abstract or complete. An abstract test purpose need only to specify what are the key events of the test. For example, an informal test purpose could be "Open the connection, do one data transfer and close the connection". A corresponding abstract, but formal test purpose would be send ConReq, send DataIn, receive DataOut, send DisconReq. A complete test purpose would integrate all the intermediate events needed to fulfil the sequence:

send ConReq, receive ConAck, send DataIn, receive DataOut, send DisconReq, receive DisconAck

A complete test purpose specifies one by one all the events needed for the test success. Test purposes generated automatically by TestComposer are 
complete in this sense. Complete test purposes are important with respect to TestPlayer, as a complete test purpose corresponds to the notion of test case used by TestPlayer.

Thus we can consider the first phase of TestComposer as a possible frontend of TestPlayer. Starting from an SDL specification describing the IUT behavior, the user can generate test cases in tMSC using TestComposer, and possibly complete the test cases by hand. Then using TestPlayer, he can execute the tMSC test cases on the IUT.

The IUT code needs not be generated from the same SDL specification, as long as the signals and their signature remain the same as it is in the SDL specification used by TestComposer. In that case, this SDL specification can be considered as the test specification, which can be developed independently and in parallel to the SDL specification meant for code generation.

But the same SDL specification can also be used, with benefits. In theory, as the tests and the code come from the same source, if an error is present in the specification, then it will be present in both the code and the tests, and the validation will declare as ok a faulty implementation. In effect, the only thing really tested would be the code generator.

In practical cases however, some of the IUT is generated from SDL, but other parts of the IUT are external libraries, parts of real time operating systems, databases. Very often a scheduling monitor is added, which can change dramatically the code behavior with respect to the specification. In this context, it makes sense to generate test case from the same source as code.

Last but not least, extensive validation campaign needed for certification purposes generally require a high, up to one hundred per cent, coverage of the code by the tests. In this context, working with the same specification makes even more sense, as the structure of the SDL and of the generated code will match. Test purposes generated by TestComposer correspond to the structural coverage of the SDL specification, so by derivation, correspond also to some extent to the structural coverage of the code.

\section{CONCLUSIONS}

We have presented TestPlayer, a tool for edition and execution of test cases written with the tMSC language. TMSC, being a subset of MSC, brings easy visualization and edition of test cases. Among its weak points, one can consider its restriction to deterministic test cases. One can not specify Inconclusive verdicts as in TTCN, these verdicts being one way to handle the IUT non-determinism during the test. However, most of the designers of 
safety critical systems are loath to deal with non-deterministic systems, especially in a context of strong certification requirements, such as in aeronautics or transport. For them, MSC are perfectly adequate for the expression of the behavior of a test case.

The architecture of the toolbox is such that it can be rapidly customized to work on different platforms (operating systems, network protocols, etc.) and different target systems. An originality point of our toolbox is the use of a second language for describing tests, TDL, which allows a more precise description than what is possible with MSCs, closer to the power of TTCN. This power of expression is provided at a low cost: TDL is a mere extension of TCL with a small learning curve.

Future developments around our tool include the industrialization of the tool by Verilog. We plan also to keep up with the future versions of the supported standards (SDL and MSC) which are both due at the end of 1999, and which will bring many substantial changes, some of them mentioned in the paper.

\section{ACKNOWLEDGEMENTS}

We wish to thank Rodolphe Arthaud who brought the initial momentum and many ideas to this project.

\section{REFERENCES}

1. ISO/IEC International Standard 9646-3. OSI-Open Systems Interconnection, Information Technology - Open Systems Interconnection Conformance Testing Methodology and Framework - Part 3: The Tree and Tabular Combined Notation (TTCN), 1992.

2. ISO/IEC International Standard 9646-1/2/3. OSI-Open Systems Interconnection, Information Technology - Open Systems Interconnection Conformance Testing Methodology and Framework, 1992.

3. J. Grabowsky and T. Walter. Visualisation of TTCN test cases by MsCs. In Proceedings of the $1^{\text {st }}$ Workshop of the SDL Forum Society on SDL and MSC, Informatik Bericht Nr. 104, Humboldt Universität Berlin, 1998.

4. Jens Grabowsky. Test Case Generation and Test Case Specification with Message Sequence Charts, Ph.D. Thesis. Universität Bern, 1994.

5. A. Kerbrat, T. Jéron, and R. Groz. Automated test generation from SDL specifications. In Proceedings of the SDL Forum '99. Elsevier Science (North Holland), 1999.

6. ITU-T Recommendation X-680. Abstract Syntax Notation One (ASN.1): Specification of Basic Notation, 1994.

7. ITU-T Recommendation Z.100. Specification and Description Language, 1996.

8. ITU-T Recommendation Z.120. Message Sequence Charts, 1996.

9. VERILOG. ObjectGEODE Reference Manuals. Technical Report, VERILOG, 1996.

10. VERILOG. TestPlayer Reference Manuals. Technical Report, VERILOG, 1999 